\title{
Generalized Singular Value Decomposition with Iterated Tikhonov Regularization
}

\author{
Alessandro Buccini ${ }^{\mathrm{a}}$, Mirjeta Pasha ${ }^{\mathrm{a}}$, Lothar Reichel ${ }^{\mathrm{a}}$ \\ ${ }^{a}$ Department of Mathematical Sciences, Kent State University, Kent, OH 44242, USA.
}

\begin{abstract}
Linear discrete ill-posed problems arise in many areas of science and engineering. Their solutions are very sensitive to perturbations in the data. Regularization methods try to reduce the sensitivity by replacing the given problem by a nearby one, whose solution is less affected by perturbations. This paper describes how generalized singular value decomposition can be combined with iterated Tikhonov regularization and illustrates that the method so obtained determines approximate solutions of higher quality than the more commonly used approach of pairing generalized singular value decomposition with (standard) Tikhonov regularization. The regularization parameter is determined with the aid of the discrepancy principle. This requires the application of a zero-finder. Several zero-finders are compared.
\end{abstract}

Keywords: Ill-posed problem, iterated Tikhonov, GSVD, zero-finder.

\section{Introduction}

We consider linear least-squares problems of the form

$$
\min _{x \in \mathbb{R}^{n}}\|A x-b\|
$$

where the matrix $A \in \mathbb{R}^{m \times n}$ has singular values that gradually decay to zero with no significant gap. In particular, $A$ may be rank deficient. Throughout this paper $\|\cdot\|$ stands for the Euclidean vector norm. We will in our discussions assume that $m \geq n$, but this constraint easily can be removed. The data vector $b \in \mathbb{R}^{m}$ is assumed to be contaminated by an unknown error $e \in \mathbb{R}^{m}$ that may stem from measurement or discretization inaccuracies.

Email addresses: abuccini@kent.edu (Alessandro Buccini), mpasha1@kent.edu (Mirjeta Pasha), reichel@math.kent.edu (Lothar Reichel) 
Least-squares problems with a matrix of this kind are referred to as linear discrete ill-posed problems. They arise, for instance, when discretizing Fredholm integral equations of the first kind; see [9, 13].

Let $b_{\text {true }}$ denote the unknown error-free vector such that

$$
b=b_{\text {true }}+e .
$$

We will assume that the vector $b_{\text {true }}$ is in the range of $A$ and that a bound $\delta$ for the error norm,

$$
\|e\| \leq \delta
$$

is known. This allows the determination of a regularization parameter with the aid of the discrepancy principle; see below. However, the method described for solving (1) also can be used, with some modifications, when these requirements are not satisfied.

We would like to determine an accurate approximation of the solution $x_{\text {true }}$ of (1) with $b$ replaced by $b_{\text {true }}$. It can be expressed as $x_{\text {true }}=A^{\dagger} b_{\text {true }}$, where $A^{\dagger}$ denotes the Moore-Penrose pseudoinverse of $A$. Due to error $e$ in $b$ and the presence of many singular values of $A$ close to the origin, the solution $A^{\dagger} b$ of (1) generally is not a meaningful approximation of $x_{\text {true }}$.

To circumvent this difficulty, one typically replaces the minimization problem (1) by a related problem, whose solution is less sensitive to the error in $b$. This replacement is referred to as regularization. Tikhonov regularization replaces (1) by a penalized least-squares problem of the form

$$
\min _{x \in \mathbb{R}^{n}}\left\{\|A x-b\|^{2}+\mu\left\|L\left(x-x^{(0)}\right)\right\|^{2}\right\}
$$

for some vector $x^{(0)}$, that may be an available approximation of $x_{\text {true }}$. If no approximation of $x_{\text {true }}$ is known, then one may choose $x^{(0)}=0$. The matrix $L \in \mathbb{R}^{p \times n}$ is referred to as a regularization matrix. Common choices of $L$ include the identity matrix $I$ or a discrete approximation of a differential operator. We require that $L$ satisfies

$$
\mathcal{N}(A) \cap \mathcal{N}(L)=\{0\}
$$

where $\mathcal{N}(M)$ denotes the null space of the matrix $M$. Then the Tikhonov minimization problem (4) has the unique solution

$$
x_{\mu}=\left(A^{T} A+\mu L^{T} L\right)^{-1}\left(A^{T} b+L^{T} L x^{(0)}\right)
$$

for any (fixed) $\mu>0$. Here and throughout this paper, the superscript ${ }^{T}$ denotes transposition. The regularization parameter $\mu>0$ determines the 
sensitivity of $x_{\mu}$ to the error $e$ in $b$, and how close $x_{\mu}$ is to the desired vector $x_{\text {true }}$.

Many methods for determining a suitable value of the regularization parameter, including the discrepancy principle, the L-curve criterion, and generalized cross validation, require repeated solution of (4) for several values of $\mu$; see, e.g., $[10,13,15,17]$. When the matrices $A$ and $L$ are of small to moderate size, one therefore often first computes the generalized singular value decomposition (GSVD) of the matrix pair $\{A, L\}$. Substituting this decomposition into (4) transforms the minimization problem into a problem with diagonal matrices, which makes repeated solution of the transformed problem for a sequence of $\mu$-values inexpensive, once the GSVD has been computed.

This paper proposes to replace Tikhonov regularization (4) by iterated Tikhonov regularization. The additional computational effort required by iterated Tikhonov regularization is negligible in comparison with the work demanded to compute the GSVD of the matrix pair $\{A, L\}$. Computed results reported in Section 4 show this approach to determine approximations of $x_{\text {true }}$ of higher quality than (standard) Tikhonov regularization (4). We remark that iterated Tikhonov regularization is a well-known regularization technique when $L=I$; see, e.g., Engl et al. [9]. The method has only recently been applied and analyzed when $L$ is a more general regularization matrix $[5,6,14]$. The latter references are concerned with large-scale problems. It is the purpose of the present paper to discuss the application of iterated Tikhonov regularization with a general regularization matrix to problems of small to moderate size, for which it is feasible to compute the GSVD of the matrix pair $\{A, L\}$.

The organization of this paper is as follows. Section 2 describes iterated Tikhonov regularization and the use of the GSVD to reduce the computational effort in every iteration step. The computation of a value of the regularization parameter by the discrepancy principle is discussed in Section 3 , where also the choice of zero-finders is considered. Computed examples are presented in Section 4 and concluding remarks can be found in Section 5.

\section{Iterated Tikhonov regularization based on the GSVD}

Let $x^{(0)}=x_{\mu}$ be an available approximation of $x_{\text {true }}$, such as a solution of (4) for some $\mu>0$. Define $r^{(0)}=b-A x^{(0)}$ and $h=x-x^{(0)}$. Then (4) can be written as

$$
\min _{h \in \mathbb{R}^{n}}\left\{\left\|A h-r_{0}\right\|^{2}+\mu\|L h\|^{2}\right\}
$$


Thus, $h$ provides an approximation of the error $x_{\text {true }}-x_{0}$. An improved approximation of $x_{\text {true }}$ is given by $x^{(1)}=x^{(0)}+h$. Repeated application of this process defines the iterated Tikhonov method:

for $k=0,1, \ldots$ do

1. Compute $r^{(k)}=b-A x^{(k)}$.

2. Solve

$$
\min _{h \in \mathbb{R}^{n}}\left\{\left\|A h-r^{(k)}\right\|^{2}+\mu^{(k)}\|L h\|^{2}\right\}
$$

to obtain $h^{(k)}$.

3. Update $x^{(k+1)}=x^{(k)}+h^{(k)}$.

In Step $2, \mu^{(0)}, \mu^{(1)}, \ldots$ denotes a sequence of positive regularization parameters. Several choices are discussed and analyzed in $[4,5,6,14]$. A specific choice will be considered in Section 3 .

The iterations for the iterated Tikhonov method can be expressed compactly in the form

$$
x^{(k+1)}=x^{(k)}+\left(A^{T} A+\mu^{(k)} L^{T} L\right)^{-1} A^{T}\left(b-A x^{(k)}\right), \quad k=0,1, \ldots .
$$

The computations required for the iterated Tikhonov method can be simplified by using the GSVD of the matrix pair $\{A, L\}$, which is given by

$$
A=U \Sigma Y^{T}, \quad L=V \Lambda Y^{T},
$$

where the matrices $U \in \mathbb{R}^{m \times m}$ and $V \in \mathbb{R}^{p \times p}$ are orthogonal, the matrix $Y \in \mathbb{R}^{n \times n}$ is nonsingular, and

$$
\begin{aligned}
\Sigma & =\operatorname{diag}\left(\sigma_{1}, \sigma_{2}, \ldots, \sigma_{p}, 1,1, \ldots, 1\right) \in \mathbb{R}^{m \times n} \\
\Lambda & =\left(\operatorname{diag}\left(\lambda_{1}, \lambda_{2}, \ldots, \lambda_{p}\right), 0,0, \ldots, 0\right) \in \mathbb{R}^{p \times n}
\end{aligned}
$$

where we assume that $1 \leq p \leq n$ and $(0,0, \ldots, 0)$ represents $n-p$ zero vectors of size $p \times 1$. We comment on below how this restriction can be removed. Thus, $\Sigma$ is a diagonal, possibly rectangular matrix, and $\Lambda$ consists of a leading $p \times p$ diagonal matrix to which $n-p$ columns of zeros are appended. The $p$ first entries of $\Sigma$ and $\Lambda$ are ordered according to

$$
0 \leq \sigma_{1} \leq \sigma_{2} \leq \ldots \leq \sigma_{p} \leq 1, \quad 1 \geq \lambda_{1} \geq \lambda_{2} \geq \ldots \geq \lambda_{p} \geq 0,
$$

and satisfy $\sigma_{i}^{2}+\lambda_{i}^{2}=1$ for $1 \leq i \leq p$; see Golub and Van Loan [11, Sections 8.7.4 and 6.1.6] and Bai and Demmel [2,3] for properties of the GSVD and 
its computation. Modifications of the GSVD have been described in [7, 8]. The quotients $\sigma_{i} / \lambda_{i}, 1 \leq i \leq p$, are commonly referred to as generalized singular values of the matrix pair $\{A, L\}$. They are the singular values of $A L^{-1}$ if the matrix $L$ is square and invertible.

For completeness, we briefly discuss the case where $L \in \mathbb{R}^{p \times n}$ with $p>n$. Consider the "reduced" QR factorization $L=Q R$, where $Q \in \mathbb{R}^{p \times n}$ has orthonormal columns and $R \in \mathbb{R}^{n \times n}$ is upper triangular. We may then replace $L$ by $R$ in both Tikhonov and iterated Tikhonov regularization without affecting the computed approximate solutions.

Equation (5) can be expressed as

$$
\left(A^{T} A+\mu^{(k)} L^{T} L\right) x^{(k+1)}=A^{T} b+\mu^{(k)} L^{T} L x^{(k)} .
$$

Substituting (6) into (7) yields

$$
\left(Y \Sigma^{T} \Sigma Y^{T}+\mu^{(k)} Y \Lambda^{T} \Lambda Y^{T}\right) x^{(k+1)}=Y \Sigma^{T} U^{T} b+\mu^{(k)} Y \Lambda^{T} \Lambda Y^{T} x^{(k)} .
$$

Since the matrix $Y$ is nonsingular, we may multiply the above equation from the left by $Y^{-1}$. Then letting $z^{(k)}=Y^{T} x^{(k)}$ gives

$$
\left(\Sigma^{T} \Sigma+\mu^{(k)} \Lambda^{T} \Lambda\right) z^{(k+1)}=\Sigma^{T} U^{T} b+\mu^{(k)} \Lambda^{T} \Lambda z^{(k)},
$$

i.e.,

$$
z^{(k+1)}=\left(\Sigma^{T} \Sigma+\mu^{(k)} \Lambda^{T} \Lambda\right)^{-1}\left(\Sigma^{T} U^{T} b+\mu^{(k)} \Lambda^{T} \Lambda z^{(k)}\right) .
$$

In view of that the matrices $\Sigma$ and $\Lambda$ are diagonal, the iterations (8) can be carried out inexpensively.

\section{The discrepancy principle and zero-finders}

The choice of the regularization parameter is important and many methods have been proposed in the literature; see, e.g., $[10,13,15,17]$ and references therein. We will apply the discrepancy principle in the present paper. It prescribes that a value of the regularization parameter $\mu^{(k)}$ be determined such that the associated approximate solution $x^{(k+1)}$ of $(7)$ satisfies

$$
\left\|A x^{(k+1)}-b\right\|^{2}=(\eta \delta)^{2} .
$$

We obtain from (7) that for $k \geq 1$ and $\mu^{(k)}>0$,

$$
x^{(k+1)}=\left(A^{T} A+\mu^{(k)} L^{T} L\right)^{-1}\left(A^{T} b+\mu^{(k)} L^{T} L x^{(k)}\right) .
$$


Substituting this expression for $x^{(k+1)}$ into (9), using the GSVD (6), and letting $\hat{b}=U^{T} b$ and $z^{(k)}=Y^{T} x^{(k)}$ yields

$$
\begin{aligned}
\left\|A x^{(k+1)}-b\right\|^{2} & =\left\|\Sigma\left(\Sigma^{T} \Sigma+\mu^{(k)} \Lambda^{T} \Lambda\right)^{-1}\left(\Sigma^{T} \hat{b}+\mu^{(k)} \Lambda^{T} \Lambda z^{(k)}\right)-\hat{b}\right\|^{2} \\
& \geq \sum_{j=1}^{p}\left(\frac{\hat{b}_{j} \lambda_{j}^{2}-\sigma_{j} z_{j}^{(k)} \lambda_{j}^{2}}{\frac{1}{\mu^{(k)}} \sigma_{j}^{2}+\lambda_{j}^{2}}\right)^{2}
\end{aligned}
$$

where $\hat{b}=\left[\hat{b}_{1}, \ldots, \hat{b}_{m}\right]^{T}$ and $z^{(k)}=\left[z_{1}^{(k)}, \ldots, z_{n}^{(k)}\right]^{T}$, and the inequality (10) is obtained by ignoring terms with index $j$ larger than $p$.

For reasons explained below, we make the change of variables $\beta=1 / \mu^{(k)}$. The right-hand side of (10) then defines the function

$$
\Phi(\beta)=\sum_{j=1}^{p}\left(\frac{\hat{b}_{j} \lambda_{j}^{2}-\sigma_{j} z_{j}^{(k)} \lambda_{j}^{2}}{\beta \sigma_{j}^{2}+\lambda_{j}^{2}}\right)^{2} .
$$

Thus,

$$
\Phi(\beta) \leq\left\|A x^{(k+1)}-b\right\|^{2}, \quad \beta=1 / \mu^{(k)} .
$$

Note that $\Phi(\beta)$ can be evaluated inexpensively for different $\beta$-values, because $p$ is of small to moderate size in applications of interest.

We seek to determine $\beta>0$ so that

$$
\Phi(\beta)=(\eta \delta)^{2} .
$$

In view of (11) and the fact that $\Phi(\beta)$ is monotonically decreasing, see below, this implies that we possibly over-regularize slightly, i.e., we determine a parameter $\mu^{(k)}=1 / \beta$ that may be slightly larger than required to satisfy (9).

It is straightforward to compute the first and second derivatives

$$
\begin{aligned}
\Phi^{\prime}(\beta) & =\sum_{j=1}^{p} \frac{-2 \sigma_{j}^{2}\left(\hat{b}_{j} \lambda_{j}^{2}-\sigma_{j} z_{j}^{(k)} \lambda_{j}^{2}\right)^{2}}{\left(\beta \sigma_{j}^{2}+\lambda_{j}^{2}\right)^{3}}, \\
\Phi^{\prime \prime}(\beta) & =\sum_{j=1}^{p} \frac{6 \sigma_{j}^{4}\left(\hat{b}_{j} \lambda_{j}^{2}-\sigma_{j} z_{j}^{(k)} \lambda_{j}^{2}\right)^{2}}{\left(\beta \sigma_{j}^{2}+\lambda_{j}^{2}\right)^{4}} .
\end{aligned}
$$


Letting $\beta \searrow 0$ in the above expressions gives

$$
\begin{aligned}
\Phi(0) & =\sum_{j=1}^{p}\left(\hat{b}_{j}-\sigma_{j} z_{j}^{(k)}\right)^{2}, \\
\Phi^{\prime}(0) & =\sum_{j=1}^{p} \frac{-2 \sigma_{j}^{2}\left(\hat{b}_{j}-\sigma_{j} z_{j}^{(k)}\right)^{2}}{\lambda_{j}^{2}} \\
\Phi^{\prime \prime}(0) & =\sum_{j=1}^{p} \frac{6 \sigma_{j}^{4}\left(\hat{b}_{j}-\sigma_{j} z_{j}^{(k)}\right)^{2}}{\lambda_{j}^{4}} .
\end{aligned}
$$

Generally, $\Phi^{\prime}(\beta)<0$ and $\Phi^{\prime \prime}(\beta)>0$ for $\beta \geq 0$. It follows that the function $\Phi(\beta)$ is monotonically decreasing and convex. Therefore, if equation (12) has a solution, then the solution is unique. There is a bounded solution when $\Phi(0)>(\eta \delta)^{2}$, which is true for most reasonable problems. We remark that the function $\mu \rightarrow \Phi(1 / \mu)$ is not guaranteed to be convex. For this reason we make the change of variables $\beta=1 / \mu$.

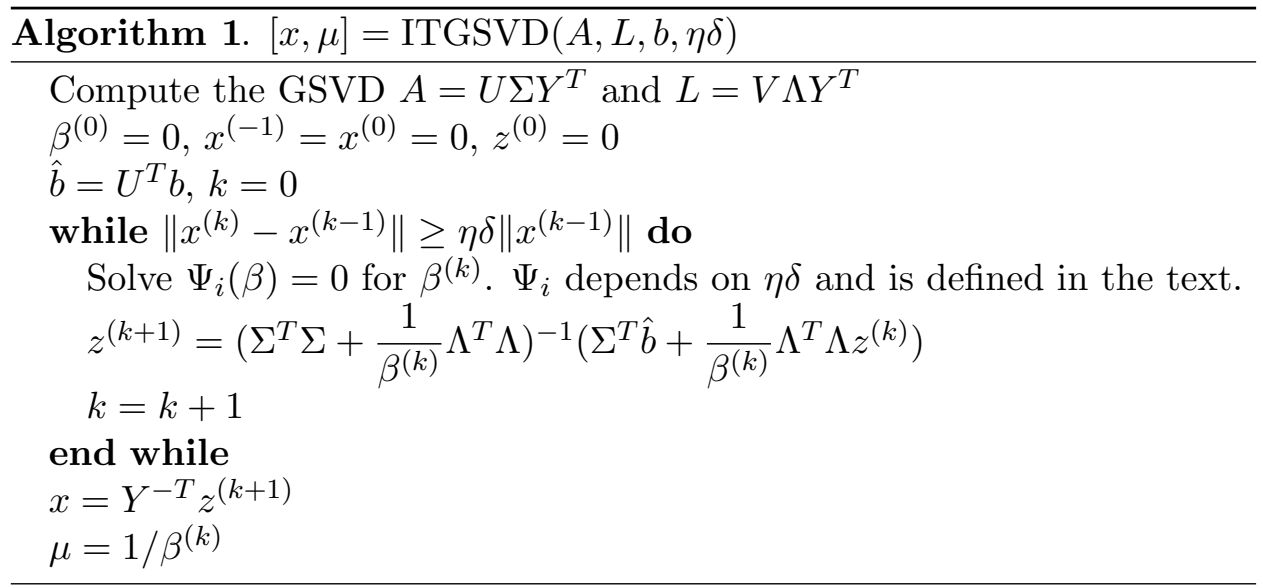

The proposed GSVD-based iterated Tikhonov method is described by Algorithm 1. After having computed the GSVD of the matrix pair $\{A, L\}$, a sequence of transformed approximate solutions $z^{(1)}, z^{(2)}, \ldots$ of (1) is generated. The regularization parameter sequence $\mu^{(1)}=1 / \beta^{(1)}, \mu^{(2)}=$ $1 / \beta^{(2)}, \ldots$ defined by the algorithm is such that each vector $x^{(k+1)}=$ $Y^{-T} z^{(k+1)}$ satisfies

$$
\left\|A x^{(k+1)}-b\right\|^{2} \geq \Phi\left(\beta^{(k)}\right) \approx(\eta \delta)^{2} .
$$

The iterations are terminated when two consecutive iterates are sufficiently close. Therefore, the last relation above is not guaranteed to be an equality. 
We compare three zero-finders for the solution of $\Phi(\beta)=(\eta \delta)^{2}$ :

(i) Newton's method applied to determining a zero of $\Psi_{1}(\beta)=\Phi(\beta)-$ $(\eta \delta)^{2}$

(ii) Newton's method applied to determining a zero of $\Psi_{2}(\beta)=1 / \Phi(\beta)-$ $1 /(\eta \delta)^{2}$, and

(iii) a cubically convergent zero-finder applied to the solution $\Psi_{1}(\beta)=0$. This zero-finder is described in [18].

While Newton's method requires the evaluation of $\Phi(\beta)$ and its first derivative, the zero-finder [18] also demands computation of the second derivative.

Since the function $\Psi_{1}(\beta)$ is monotonically decreasing and strictly convex for $\beta>0$, Newton's method gives monotonic and quadratic convergence of the iterates $\beta^{(k)}$ if the initial iterate is smaller than or equal to the solution of (12). Hence, for $\beta^{(0)}=0$, the method does not have to be safe-guarded to guarantee convergence. However, computed examples reported in [18] show that, with this initial iterate, Newton's method may require fairly many iterations. It therefore may be beneficial to use a cubically convergent zero-finder. This is illustrated in [18] for standard Tikhonov regularization. Reinsch [19] points out that Newton's method applied to the computation of a zero $\Psi_{2}$ may require fewer steps to satisfy a convergence criterion than Newton's method applied to the determination of a zero of $\Psi_{1}$, because $\Psi_{2}$ sometimes can be approximated more accurately by a linear function in the vicinity of the zero than $\Psi_{1}$. We therefore include Newton's method applied to computing a zero of $\Psi_{2}$ in our comparison.

\section{Numerical examples}

This section presents a few computed examples. The main goal of this section is to show that iterated Tikhonov regularization combined with the GSVD is competitive with (standard) Tikhonov regularization based on the GSVD. We will refer to these methods as ITikGSVD and TikGSVD, respectively. The examples illustrate that the iterations in the ITikGSVD method do not increase the computing time significantly over the computing time required by TikGSVD, but they improve the quality of the computed approximation of $x_{\text {true }}$. Typically, a few iterations in ITikGSVD suffice to improve the quality of the computed approximate solution significantly. The examples also show the performance of the zero-finders described in Section 
3. In all examples, we use $\eta=1.01$ in (12) and the initial approximate solution $x^{(0)}=0$ for the iterations. The regularization matrix is the tridiagonal matrix

$$
L=\left(\begin{array}{cccccc}
-1 & 2 & -1 & & & \\
& \cdot & \cdot & \cdot & & \\
& & \cdot & \cdot & \cdot & \\
& & & -1 & 2 & -1
\end{array}\right) \in \mathbb{R}^{(n-2) \times n},
$$

which is a scaled discretization of the second derivative operator at equidistant points in one space-dimension. The examples stem from the MATLAB package Regularization Tools by Hansen [12]. All examples are obtained by discretizing a Fredholm integral equation of the first kind with a square integrable kernel,

$$
\int_{a}^{b} K(s, t) x(t) d t=g(s), \quad c \leq s \leq d,
$$

where the right-hand side function $g$ and the kernel $K$ are given, and we seek to determine the solution $x$. Discretization is carried out by Galerkin or Nystrom methods and yield a linear discrete ill-posed problem (1). MATLAB functions from [12] determine discretizations $A \in \mathbb{R}^{n \times n}$ of the integral operators and scaled discrete approximations $x_{\text {true }} \in \mathbb{R}^{n}$ of the solution $x(t)$ of (14). We let $b_{\text {true }}=A x_{\text {true }}$. In all examples we fix $n=400$.

The error vector $e \in \mathbb{R}^{400}$ is modeled by white Gaussian noise and we refer to the ratio

$$
\sigma=\frac{\|e\|}{\left\|b_{\text {true }}\right\|}
$$

as the noise level. The error contaminated right-hand side $b$ is obtained by (2). The relative reconstruction error of the computed solution $x_{\mu^{(k)}}$ is given by

$$
\operatorname{RRE}\left(x_{\mu^{(k)}}\right)=\frac{\left\|x_{\mu^{(k)}}-x_{\text {true }}\right\|}{\left\|x_{\text {true }}\right\|} .
$$

Example 4.1. We first consider the problem implemented by the code deriv2 from [12]. The kernel, solution, and right-hand side of (14) are given by

$$
\begin{aligned}
K(s, t) & = \begin{cases}s(t-1), & s<t, \\
t(s-1), & s \geq t,\end{cases} \\
x(t) & =\exp (t), \\
g(s) & =\exp (s)+(1-\exp (1)) s-1 .
\end{aligned}
$$




\begin{tabular}{ccccc} 
& Iterations & RRE & Time & Time GSVD \\
\hline ITikGSVD & 5 & 0.0057 & 0.194 & 0.130 \\
TikGSVD & & 0.0396 & 0.180 & 0.130
\end{tabular}

Table 1: Example 4.1: ITikGSVD results are shown in the first row and results for TikGSVD in the second row. The column labeled "Iterations" shows the number of iterations required by ITikGSVD. The times are in seconds. The last column shows the time needed for calculating the GSVD only. The noise level is $\sigma=0.001$.

\begin{tabular}{ccccc} 
& Iterations & RRE & Time & Time GSVD \\
\hline ITikGSVD & 4 & 0.016 & 0.185 & 0.144 \\
TikGSVD & & 0.039 & 0.184 & 0.144
\end{tabular}

Table 2: Example 4.1: This table differs from Table 1 in that the noise level is $\sigma=0.01$.

and $a=c=0, b=d=1$. The kernel $K$ is the Green's function for the second derivative.

Tables 1 and 2 show the RRE to drop significantly by carrying out a few iterations in the ITikGSVD method. The computing time is dominated by the time required to calculate the GSVD of the matrix pair $\{A, L\}$. The ITikGSVD method is seen to require very little more computing time than the TikGSVD method. Figure 1 shows the computed solutions determined by ITikGSVD and TikGSVD. The former is seen to furnish a better approximation of $x_{\text {true }}$.

Example 4.2. The test problem baart from [1] is a discretization of an integral equation (14) with the kernel and right-hand side function given by

$$
K(s, t)=\exp (s \cos (t)), \quad g(s)=2 \frac{\sin (s)}{s},
$$

and with $0 \leq s \leq \pi / 2$ and $0 \leq t \leq \pi$. The solution is given by $x(t)=\sin (t)$.

Using the code baart from [12], we discretize (14) by a Galerkin method with 400 orthonormal box functions as test and trial functions. This generates the matrix $A \in \mathbb{R}^{400 \times 400}$ and the solution $x_{\text {true }}$, which is a scaling of $x(t)$. We determine the right-hand side vector $b_{\text {true }}=A x_{\text {true }}$ and the errorcontaminated right-hand side vector $b$ like in Example 4.1. The matrix $A$ is numerically singular.

Figure 2 shows the computed solutions and is analogous to Figure 1. The ITikGSVD method is seen to give the most accurate approximation of $x_{\text {true }}$. Tables 3 and 4 are similar to the tables shown in Example 4.1. They show the ITikGSVD method to give more accurate approximations of $x_{\text {true }}$ than TikGSVD and only require insignificantly more computing time. 


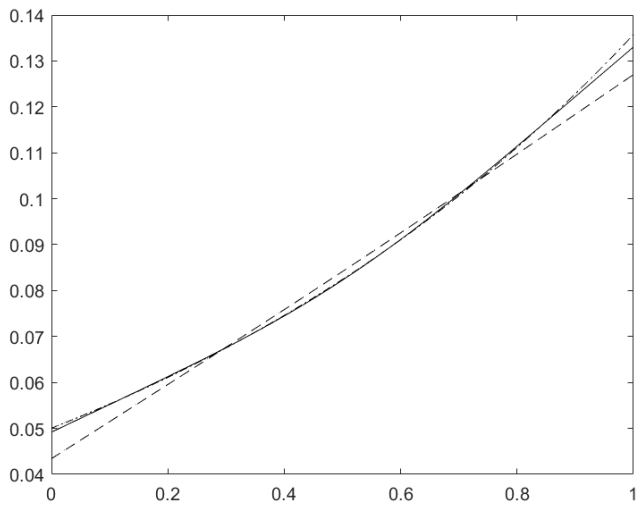

Figure 1: Example 4.1: The dash-dotted curve shows $x_{\text {true }}$, the solid curve displays the approximation calculated by ITikGSVD, and the dashed curve depicts the approximation determined by TikGSVD.

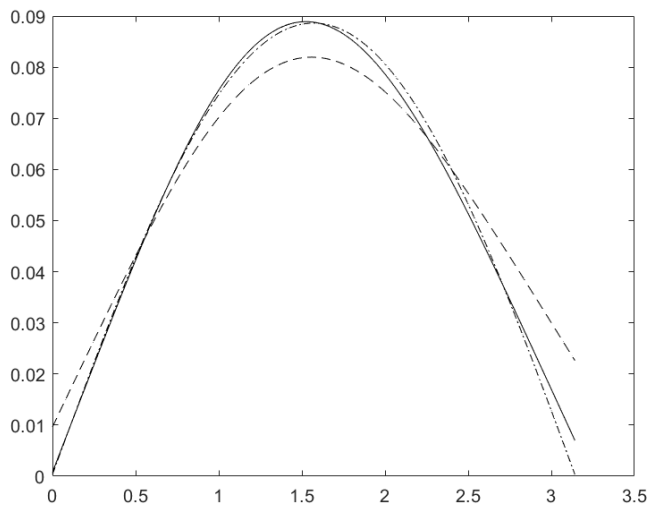

Figure 2: Example 4.2: The dash-dotted curve shows $x_{\text {true }}$, the solid curve displays the approximation calculated by ITikGSVD, and the dashed curve depicts the approximation determined by TikGSVD. 


\begin{tabular}{ccccc} 
& Iterations & RRE & Time & Time GSVD \\
\hline ITikGSVD & 3 & 0.0271 & 0.149 & 0.119 \\
TikGSVD & & 0.0314 & 0.148 & 0.119
\end{tabular}

Table 3: Example 4.2: ITikGSVD results are shown in the first row and TikGSVD results in the second row. "Iterations" shows the number of iterations required by ITikGSVD. The times are in seconds. The last column shows the time required for calculating the GSVD only. The noise level is $\sigma=0.001$.

\begin{tabular}{ccccc} 
& Iterations & RRE & Time(s) & Time(s) GSVD \\
\hline ITikGSVD & 5 & 0.037 & 0.142 & 0.123 \\
TikGSVD & & 0.255 & 0.141 & 0.123
\end{tabular}

Table 4: Example 4.2: This table differs from Table 3 in that the noise level is $\sigma=0.01$.

Example 4.3. In Examples 4.1 and 4.2, we carried out the computations with Algorithm 1 and computed the zero of the function $\Psi_{1}$ by the method in [18]. The present example compares the performance of the zero-finders considered in Section 3. The problem solved is the same as in Example 4.2, but the noise level differs. For the present example it is $\sigma=0.05$. Moreover, the computations are carried out on a different computer than the one used for Examples 4.1 and 4.2. Therefore, the timings in Table 5 cannot be compared with those reported in the previous tables. The RRE and the number of iterations are not affected by the change of computer.

\begin{tabular}{cccccc} 
& \multicolumn{2}{c}{ Iterations } & Tikhonov & $\operatorname{RRE}\left(x_{\mu^{(k)}}\right)$ & Time \\
Zero-finder & First & Total & & & \\
\hline Newton for $\Psi_{1}$ & 12 & 16 & 4 & 0.038858 & 0.2102 \\
Newton for $\Psi_{2}$ & 10 & 12 & 2 & 0.038857 & 0.1831 \\
Method [18] & 8 & 10 & 3 & 0.038857 & 0.1532
\end{tabular}

Table 5: Example 4.3: Comparison of zero-finders for computing the regularization parameter.

Table 5 compares the zero-finders of Section 3. The number of steps are shown in the columns labeled "Iterations". The column labeled "First" displays the number of steps required to determine the first regularization parameter, with initial approximation $\beta^{(0)}=0$. This is the number of steps required by TikGSVD. The column labeled "Total" shows the total number of steps of each zero-finder required for all iterations in the ITikGSVD method. The number of iterations of the ITikGSVD method is displayed in the column "Tikhonov". Since the regularization parameters $\mu^{(k)}, k=1,2, \ldots$ are 
close, only one or two steps of the zero-finder are required for each iteration with the ITikGSVD method. The column labeled "RRE" shows the relative errors in the computed solutions determined by the ITikGSVD method. The errors are seen to be essentially independent of the zero-finder used. The last column displays the times required. It shows the cubically convergent zero-finder discussed in [18] to require the fewest iterations and also result in the fastest implementation of ITikGSVD. For all zero-finders, the most iterations are required to compute the initial regularization parameter used by ITikGSVD.

Example 4.4. This example illustrates the performance of the ITikGSVD method when applied to the restoration of an image. We use the MATLAB code blur from [12] with default parameter values band $=3$ and sigma $=0.7$ to generate the blurring matrix $A \in \mathbb{R}^{1024 \times 1024}$, which models Gaussian blur, and a test image made up of $32 \times 32$ pixels. The pixels are stored columnwise in the vector $x_{\text {true }}$. The test image is displayed in Figure 3(a). The vector $b_{\text {true }}=A x_{\text {true }}$ represents the blurred, but noise-free image, to which we add $5 \%$ Gaussian noise. This yields the blur- and noise-contaminated image displayed in Figure 3(b). The vector $b$ in (1) is made up of the pixels, ordered column-wise, of the latter image. Table 6 displays results for the TikGSVD and ITikGSVD methods applied to this image restoration problem. The restored image determined by ITikGSVD is shown in Figure $3(\mathrm{c})$. The restoration determined by TikGSVD looks almost the same. We therefore do not show the latter.

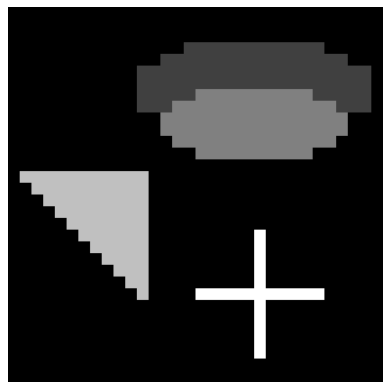

(a)

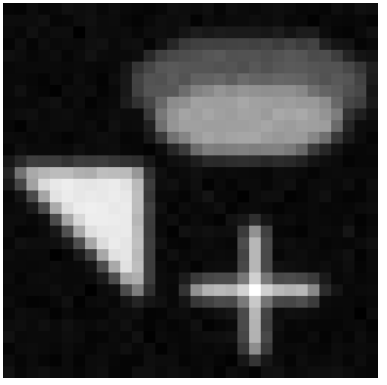

(b)

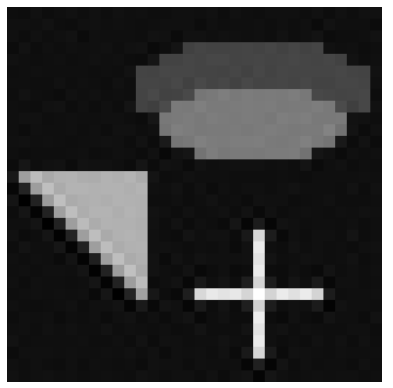

(c)

Figure 3: Blur test problem: (a) Uncontaminated image $(32 \times 32$ pixels $)$, (b) blur- and noise-contaminated image $(\sigma=0.05)$, (c) Reconstructed image by ITikGSVD.

Example 4.5. In this example we assume that $x_{\text {true }}$ is known and choose the regularization parameter $\mu$ in the ITikGSVD method so that the computed solution $x_{\mu}$ is close to $x_{\text {true }}$. This is not a practical approach 


\begin{tabular}{ccccc} 
& Iterations & RRE & Time(s) & Time(s) GSVD \\
\hline ITikGSVD & 4 & 0.0766 & 0.932 & 0.5946 \\
TikGSVD & & 0.1199 & 0.925 & 0.5946
\end{tabular}

Table 6: Example 4.4: Comparison of the RRE and CPU time in seconds for ITikGSVD and TikGSVD.

to choosing the regularization parameter. The purpose of this example is to illustrate the quality of the regularization parameter determined by the discrepancy principle. We consider the baart test problem described in Example 4.2 and generate a sequence of equidistant regularization parameter values $\mu_{k}, k=1,2, \ldots$, that are centered at the parameter value determined by the discrepancy principle. We then choose the computed solution $x_{\mu_{k}}$ that is closest to $x_{\text {true }}$. We refer to this approach to compute an approximation of $x_{\text {true }}$ as the ITikGSVDt method. Table 7 displays the error in the computed solution determined by ITikGSVDt as well as the error in the approximate solution determined by ITikGSVD. As can be expected, ITikGSVDt gives a more accurate approximation of $x_{\text {true }}$ than ITikGSVD, but the difference in quality is modest. In this example the noise level is small. For large noise levels neither ITikGSVD nor ITikGSVDt yield accurate approximations of $x_{\text {true. }}$.

\begin{tabular}{cc} 
& RRE \\
\hline ITikGSVD & 0.0271 \\
ITikGSVDt & 0.0212
\end{tabular}

Table 7: Example 4.5: The relative error in computed solutions determined by the ITikGSVD and and ITikGSVDt methods. The former determines the regularization parameter by the discrepancy pronciple, the latter determines the regularization parameter using knowledge of $x_{\text {true }}$. The noise level is $\sigma=0.001$.

\section{Conclusion}

This paper develops a new method for the solution of linear discrete illposed problems with a system matrix $A$ and a regularization matrix $L$ of small to moderate size. The method combines the GSVD method with iterated Tikhonov regularization. Computed examples show the new method to give approximate solutions of higher quality than the approximate solutions determined by combining GSVD and (standard) Tikhonov regularization. Timings show the additional computing time required by replacing standard Tikhonov regularization by iterated Tikhonov regularization to be 
negligible. The paper determines a regularization by using the discrepancy principle. This requires application of a zero-finder. A cubically convergent zero-finder described in [18] is compared with two ways to implement Newton's method and found to require fewer iterations to determine a zero with comparable accuracy.

\section{Acknowledgments}

The authors would like to thank the referees for comments. A.B. is a member of the GNCS group of INdAM that partially funded this work. The work of L.R. is partially supported by NSF grants DMS-1720259 and DMS-1729509.

\section{References}

[1] M. L. Baart, The use of auto-correlation for pseudo-rank determination in noisy ill-conditioned least-squares problems, IMA J. Numer. Anal., 2 (1982), pp. 241-247.

[2] Z. Bai, The CSD, GSVD, their applications and computation, IMA preprint 958, Institute for Mathematics and Its Applications, University of Minnesota, Minneapolis, 1992.

[3] Z. Bai and J. W. Demmel, Computing the generalized singular value decomposition, SIAM J. Sci. Comput., 14 (1993), pp. 1464-1486.

[4] M. Brill and E. Schock, Iterative solution of ill-posed problems - a survey, Model Optimization in Exploration Geophysics, 1 (1987), pp. 1737.

[5] A. Buccini, Regularizing preconditioners by non-stationary iterated Tikhonov with general penalty term, Appl. Numer. Math., 116 (2017), pp. 64-81.

[6] A. Buccini, M. Donatelli, and L. Reichel, Iterated Tikhonov regularization with a general penalty term, Numer. Linear Algebra Appl., 24 (2017), e2089 (12 pages)

[7] L. Dykes, S. Noschese, and L. Reichel, Rescaling the GSVD with application to ill-posed problems, Numer. Algorithms, 68 (2015), pp. 531-545. 
[8] L. Dykes and L. Reichel, Simplified GSVD computations for the solution of linear discrete ill-posed problems, J. Comput. Appl. Math., 225 (2013), pp. 15-27.

[9] H. W. Engl, M. Hanke, and A. Neubauer, Regularization of Inverse Problems, Kluwer, Dordrecht, 1996.

[10] C. Fenu, L. Reichel, and G. Rodriguez, GCV for Tikhonov regularization via global Golub-Kahan decomposition, Numer. Linear Algebra Appl., 23 (2016), pp. 467-484.

[11] G. H. Golub and C. F. Van Loan, Matrix Computations, 4th ed., Johns Hopkins University Press, Baltimore, 2013.

[12] P. C. Hansen, Regularization tools: A MATLAB package for analysis and solution of discrete ill-posed problems, Numer. Algorithms, 6 (1994), pp. 1-35.

[13] P. C. Hansen, Rank-Deficient and Discrete Ill-Posed Problems, SIAM, Philadelphia, 1998.

[14] G. Huang, L. Reichel, and F. Yin, Projected nonstationary iterated Tikhonov regularization, BIT Numer. Math., 56 (2016), pp. 467-487.

[15] S. Kindermann, Convergence analysis of minimization-based noise level-free parameter choice rules for linear ill-posed problems, Electron. Trans. Numer. Anal., 38 (2011), pp. 233-257.

[16] D. L. Phillips, A technique for the numerical solution of certain integral equations of the first kind, J. ACM, 9 (1962), pp. 84-97.

[17] L. Reichel and G. Rodriguez, Old and new parameter choice rules for discrete ill-posed problems, Numer. Algorithms, 63 (2013), pp. 65-87.

[18] L. Reichel and A. Shyshkov, A new zero-finder for Tikhonov regularization, BIT Numer. Math., 48 (2004), pp. 627-643.

[19] C. Reinsch, Smoothing by spline functions. II, Numer. Math., 16 (1971), pp. $541-454$.

[20] C. B. Shaw, Jr., Improvements of the resolution of an instrument by numerical solution of an integral equation, J. Math. Anal. Appl., 37 (1972), pp. 83-112. 